\title{
Temporal tuning of daily rhythms helps advanced cancer patients and cancer survivors feel better, live better, and live longer
}

This article was published in the following Dove Press journal:

ChronoPhysiology and Therapy

8 January 2016

Number of times this article has been viewed

\author{
William Hrushesky ${ }^{1-5}$ \\ James F Grutsch ${ }^{6}$ \\ Dinah Faith Q Huff' \\ Linda Tavolacci ${ }^{7}$ \\ Thomas Kazlausky \\ 'Oncology Analytics, Inc., Plantation, \\ FL, USA; ${ }^{2}$ South Carolina College of \\ Pharmacy, ${ }^{3}$ Arnold School of Public \\ Health, University of South Carolina, \\ Columbia, SC, USA; ${ }^{4} \mathrm{Hollings}$ Cancer \\ Center, ${ }^{5}$ Department of Public \\ Health Science, Medical University \\ of South Carolina, Charleston, SC, \\ USA; ${ }^{6}$ Department of Epidemiology \\ and Biostatistics, University of Illinois \\ School of Public Health, Chicago, IL, \\ USA; ${ }^{7}$ Ambulatory Monitoring, Inc., \\ Ardsley, NY, USA
}

Video abstract

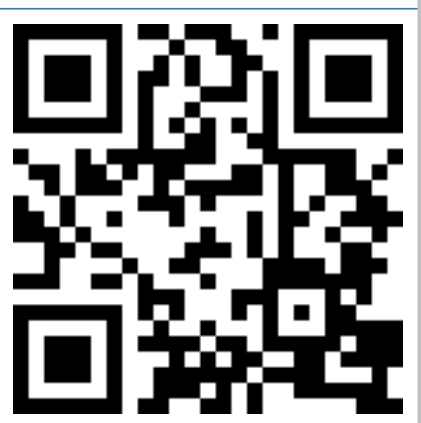

Point your SmartPhone at the code above. If you have a QR code reader the video abstract will appear. Or use: http://youtu.be/y WvailDu6s

Correspondence: William Hrushesky 12 North Cobane Terrace,

West Orange, NJ 07052, USA

Email williamhrushesky@gmail.com
Abstract: There is now little doubt that disrupted day/night (circadian) time structures are involved in the initiation and promotion of neoplastic disease. It has been established that the incidence of breast cancer, colorectal cancer, and prostate cancer is increased as a result of nocturnal exposure to light and circadian function disruption and that cancer patient survival is diminished. So the question is: what public health measures can be implemented to minimize these health hazards? In addition, untreated cancer patients experience the symptom cluster of brief, interrupted, and poor nighttime sleep; depressed mood/anxiety; daytime fatigue/lethargy; and anorexia/early satiety/diminished taste sensation - each of which is virtually pathognomonic of a disrupted circadian temporal organization. Direct measurements of patients' activities and their timing and intensity using actigraphy reveal that untreated cancer patients experience severe deterioration in the robustness (amplitude) and day-to-day phase stability of their daily rest/ activity rhythms - and one of the most personal and socially destructive results of such circadian disorientation is unplanned, unwanted, and avoidable temporal isolation from family, friends, and society. Thus, therapeutic manipulation of the circadian clock is a powerful tool for improving cancer patients' quality of life (QOL), making life more worth living and perhaps prolonging higher quality survival. We herein take what we have learned to design and execute strategies shown to be beneficial and carefully measure cancer patient benefits. Outcome measures include indices that describe our ability to enhance/maintain circadian organization and orientation, diminish the above-mentioned symptoms, and improve QOL and survival prolongation. We herein implement a suite of noninvasive, riskless and costless, largely behavior-based circadian rhythm entrainment and disturbance avoidance techniques for widespread everyday use by cancer patients and survivors together with real-time actigraphic monitoring, continuous electronic feedback, and positive reinforcement of these simple temporal tuning interventions.

Keywords: actigraphy, circadian clock, circadian rhythm, circadian therapy, nighttime sleep, daytime activity, fatigue, depression, anxiety, functional status, quality of life, survival, 24-hour activity monitoring

\section{Introduction}

The discovery that cancer initiation and progression is driven by the accumulation of mutations is one of the triumphs of 20th century biology. ${ }^{1}$ This line of research has been recently supercharged by the powerful tools of "omic" biology. Among the unaccountable number of mutations found in cancer cells, there is a mountain of evidence that 200 of the 21,000 genes making up the human genome are drivers of carcinogenesis. ${ }^{2}$ These genes often code proteins involved in cell proliferation and programmed cell death $^{3}$ and are basis for the recent flood of targeted anticancer therapies. 
Drugs targeted against the products of driver genes (ie, HER2, RAS, RAF, MEK, ERK, PI3K, AKT, and mTOR) have become the foundation of personalized medicine. ${ }^{4}$ These drugs, however, only produce a temporary reduction of several months in tumor size or growth rate. ${ }^{5}$ Today, as noted by many, it is still impossible to cure most patients with late stage carcinomas and sarcomas. ${ }^{1}$

It is going to take much more time for this piece-by-piece treatment strategy to become effective in curing patients with advanced tumors. Neoplastic disease is a Darwinian process in which mutations that drive tumor development can occur at any time throughout the life history of the tumor and its downstream colonies, and they are selected because of that fundamental survival advantage. ${ }^{6,7}$ Furthermore, the intrinsic genetic instability of late stage tumors enables neoplastic tissues to generate a near infinite number of pathways in the evolution of viable and treatment resistant cell lines. ${ }^{1,89}$ This would point to the need to develop many more drugs and have the clinical capacity to identify emergent molecular targets in near real time. It is unclear whether this treatment strategy will ever become effective in significantly prolonging a cancer patient's life at a cost that is affordable. ${ }^{1}$

\section{Circadian clocks as therapeutic targets}

Tumor cells have biochemical, molecular, and physiological weaknesses that can be leveraged, making them vulnerable to treatment. One weakness is their almost universal retention of circadian organization of the processes regulating cell proliferation, DNA repair and apoptosis, and all other fundamental functions. ${ }^{1}$ Circadian rhythms integrate, coordinate, and optimize an individual's physiological function at all levels of biological complexity, ranging from the molecular to our niche in an ecosystem. In human beings circadian rhythms synchronize and coordinate the functioning of basic human physiology, endocrinology, detoxification, cell growth, and behavior with the continuously modulating circadian environment. ${ }^{10-13}$ There are epidemiological, biological, molecular, and genetic information that show that circadian function of the cancer bearing host is disrupted soon after tumor initiation. ${ }^{14}$ Clock gene products regulating the cellular processes within cells are fundamental to carcinogenesis; cell proliferation, apoptosis, and/or DNA damage repair ${ }^{15}$ are also altered, making their tuning an important theoretical anticancer strategy. Indeed, recent research using animal strains not prone to cancer find that clock disruption can aid cancer regression by promoting intrinsic and extrinsic apoptosis..$^{16}$

\section{Clock gene mutations linked to tumor growth and spread}

Tumor development and growth are accelerated in jet lagged animals thus demonstrating the capacity of a chronically disrupted circadian rhythm to promote tumor progression, growth, and spread. ${ }^{17}$ Also, molecular epidemiology data show that mutated circadian gene products are near ubiquitous within human cancers. ${ }^{9,18,19}$ The clock gene Period or PER appears to be a premier clock gene "oncogene". The proteins the Period gene encodes are transcription factors which regulate the expression of other transcription factors and the oncogenic version of PER gene products are found in the pathways of many types of cancer. Animal experiments combining PER2 mutations with the APC gene mutations (APC protein regulates the $\beta$-catenin levels which are needed for cell adhesion) nearly doubled the tumor growth rate, compared to mice carrying only the APC mutation..$^{20}$ The normal nonmutated version of the PER2 gene product suppresses the formation of intestinal tumors in mice through a mechanism that involves the regulation of $\beta$-catenin and its downstream targets. ${ }^{20}$

\section{Circadian disruption of clock gene protein expression linked to tumor growth and spread}

Other experiments provide unique insights into the relationship between a host's circadian function and the circadian rhythms in tumor proliferation and growth. In mice with breast cancer, the production of the PER1 and PER2 gene products in their disease-free livers is high at night and low in the daytime, thus showing a stable circadian dynamic. ${ }^{21}$ These mice breast cancers also followed a circadian dynamic, but its rhythm was distorted with peaks in growth rate occurring once at night and once during each day. ${ }^{20} \mathrm{PER}$ protein expression was lower in the tumors and the time of peak expression was shifted compared to normal tissues. The expression of other clock-controlled gene products was also altered in the tumor. Reducing the expression of PER genes in the tumors both increased their growth rate and altered their usual circadian growth rhythm. This and other data demonstrate that tumors have their own internal clocks, but that they can respond to signals from the organism's clock as well. ${ }^{21}$ There are now many examples where the PER genes are involved in regulating transcription factors involved in the regulation of molecular pathways involved in tumor progression: proliferation, DNA repair, and apoptosis. ${ }^{22}$ These findings suggest the hypothesis that therapies that restore robust circadian rhythms 
could provide a significant clinical benefit for patients with advanced cancer.

The PER2 gene product's most significant antitumor mechanism of action may be its capacity to suppress the MYC transcription factor. MYC's role is to push cancer cells through the cell cycle independently of cellular, metabolic, and other restraints to cell proliferation. One of MYC's restraints is the PER2 gene product which shuts down MYC capacity to induce gene transcription. PER2's ability to inhibit MYC activity is probably why it is a tumor suppressor gene. ${ }^{1}$ However, cancer cells have other means of reducing PER gene levels that result in high MYC gene activity. ${ }^{22,23}$

MYC's capacity to push the rates of cancer proliferation into superdrive arises following the removal of PER2 gene. In many tumor cells PER 2 proteins undergo proteasome-mediated degradation via high levels of the protein encoded by the gene protein kinase casein kinase 2 . These results suggest that PER anticancer action arises from its ability to turn off MYC. In PER2's absence, MYC levels greatly rise, thereby explaining why tumors of many types all display higher levels of CSNKe than found in their normal cell equivalents. It argues that specific CSNKe inhibitors should soon be broadly tested against a large variety of human cancers. ${ }^{1,23}$ Still no clock gene related drugs are under development.

\section{Circadian disruption is a human carcinogen}

Human beings evolved to be active during daylight and to sleep at night; indeed, our nocturnal vision is very poor. Human tissues (in fact, all vertebrate tissues) organize their critical metabolic and tissue functions to specific phases of the solar day via circadian clocks, which unsurprisingly have a periodicity of a solar day and are daily tuned and retuned to this 24-hour period duration. ${ }^{10}$

The human neurologic central circadian clock is a bilateral structure found in the anterior hypothalamus of the brain known as the suprachiasmatic nuclei (SCN). This brain region controls multiple circadian rhythms at the organismal level, such as sleep, hunger, and thirst, and physiological rhythms such as blood pressure, body temperature, immune responses, and hormone production, and with it immunologic functions and pathways and tissue proliferative dynamics. ${ }^{21,24,25}$

One of outputs of the SCN is the nocturnal pulse of the chronobiotic hormone melatonin from the pineal gland. ${ }^{26}$ These pulses of melatonin synchronize many of the body's peripheral clocks and inform the body's cells that it is now the dark phase of the solar day. Distortions in the production or release of melatonin have been linked to a variety of symptoms that afflict virtually all cancer patients, such as insomnia and daytime drowsiness. Late and even early-stage cancer patients often have diminished nocturnal melatonin release, demonstrating a failing circadian organization. ${ }^{27}$

\section{Hazards of nighttime light exposure}

With the invention of artificial light during the late 19 th century, modern society inadvertently embarked on an uncontrolled public health experiment where humans for the first time during our evolution were chronically exposed to artificial light at night. ${ }^{15,28}$

Stevens has proposed that nighttime light exposure is a risk factor in the development of breast cancer. Since his proposal, a number of large prospective cohort studies have linked shift work to adverse health outcomes for a number of chronic diseases, especially cancer. The Nurses' Health Study, a massive prospective cohort epidemiological study of some 250,000 female participants, was the investigation that initially produced convincing evidence that linked shift work and the circadian disruption it universally causes to elevated cancer risk. In two very large cohorts shift workers had a markedly increased cancer burden after controlling for age, diet, body mass index (BMI), and lack of sleep. The investigators found that shift workers had lower levels of melatonin but a $36 \%-79 \%$ increased risk of breast cancer, a $35 \%$ increased risk of colorectal cancer, and a $43 \%$ increase in the risk of endometrial cancer when compared to nonshift workers. On the other hand, participants with high melatonin levels had a $41 \%$ reduction in the risk of breast cancer. $^{18,29}$

This epidemiological investigation, and many more since, has shown that nocturnal suppression of melatonin due to exposure to bright light at night was linked to breast cancer tumor growth. Subsequent cohort studies have found similar links between nocturnal exposure to light and the development of prostate and colorectal cancer. Epidemiologically, the link between circadian rhythm disruptions and cancer was established. ${ }^{30}$

The epidemiological data linking light at night and human breast cancer was established before it was confirmed in animal models. The nocturnal production of melatonin in rats was suppressed in a dose dependent manner with increasing nocturnal exposure to light. ${ }^{31}$ In a subsequent experiment, animals with human breast cancer xenografts showed accelerated tumor growth rates and decreased time to tumor onset. ${ }^{32}$ 


\section{Diet affects circadian dynamics}

More recent data uncovered how diminished production of melatonin alters the cellular physiology of cell proliferation. A common fatty acid in our diet, linoleic acid, is an excellent source of caloric energy for cells, cancer or otherwise, and it has an important signaling role in cell proliferation. At the cellular level, the uptake of linoleic acid activates a number of intracellular signaling pathways that promote cell proliferation. Melatonin reduces the cellular uptake of linoleic acid and thereby inhibits these growth-promoting pathways. ${ }^{33}$ This phenomenon has been shown to occur in human breast, head and neck, and urogenital cancers.

An investigation was done on linoleic uptake of breast cancer xenografts grown in nude mice. Mice kept on a regular light/dark schedule had rhythmic linoleic uptake in the tumors, peaking late in the day. Exposing mice to light at night resulted in replacing the rhythmic uptake of linoleic acid with a high uptake throughout the day. The tumors still grew in the presence of an intact light/dark cycle in a daily rhythm, but growth rates were dramatically increased when this cycle was disrupted or absent. ${ }^{34}$

\section{Measuring circadian rest/activity patterns in advanced cancer patients}

There have been several investigations using actigraphy to measure the sleeping behavior of cancer patients. Most of these investigations were descriptive and focused on breast cancers. Overall, these investigations have found cancer patients take many prolonged but highly disturbed daytime naps, while having many episodes of prolonged wakefulness during each night and that sleep disturbances become ever worse with continuing treatment. ${ }^{35,36}$

To illustrate the depth of quantitative data and objective data that actigraphy can provide on the extent of sleep disturbances, we summarize the results of patients with late-stage lung cancer. These results were acquired in outpatients before they underwent chemotherapy treatment. ${ }^{37}$ In addition to the actigraphy data the patients provided a self-report of their sleep quality using the Pittsburg Sleep Quality Index (PSQI), which measures seven components related to sleep. The parameters used to describe actigraphy readings also correlate with each of the PSQI measures of sleep quality. The patients' PSQI scores had much worse scores on sleep quality than population-based controls. The actigraphy data confirmed the patients self-report of poor sleep quality. The patients' mean nocturnal sleep span was 4.7 hours when compared to the adult's normal sleep span of 7-9 hours. Healthy adults take less than 20 minutes to fall asleep after going to bed, but our patients took more than twice as long..$^{38}$ Normally, adults awaken 2-6 times per night and remain awake for a total of less than 40 minutes, ${ }^{39,40}$ but our cancer patients' mean awake time during the night was 95 minutes. Daytime inactivity in our control population was 46.5 minutes, while our patients' daytime napping time was 3.5 hours/day. Finally, the patients' daily activity rhythm was severely damped in comparison to the population-based control group. ${ }^{37}$ These objectively studied cancer patients have terribly abnormal and highly symptomatic circadian daytime activity and nighttime sleep. They need help.

\section{Breaking the vicious cycle Evidence-based strategies for a healthier circadian function and how technology can help}

We currently have the ability to diminish circadian disruption and restore a more normal circadian function in patients undergoing cancer chemotherapy. Data support the combined application of 1) optimally tuned environmental synchronizers such as meal timing, and daily modulation of its caloric and carbohydrate/protein/fat composition as well as regular and optimally timed dietary stimulants; 2) properly timed and proper intensity light exposures (daily sun exposure); 3) avoidance of evening light and blue wavelength light in particular; 4) regular sleeping and waking schedules; 5) good sleep hygiene with total darkness, cool temperatures, and total silence; 6) avoidance of the administration of specific medications at specific times of day and the optimal timing and dosing of all short- or long-acting pain medications; 7) scrupulous avoidance of all long-acting soporifics; and 8) the proper timing of hormonal sleep aids or very short duration aids. Among the most important of all logical and simple strategies is the careful and total removal of all unnecessary medications and the use of clearly essential medicines. No cancer patient under active treatment needs a statin and its side effects. All but a very few should be taken off all medicines which lower blood pressure. All doses of drugs which cannot be removed should be evaluated for lowering each at least monthly. More harm is done to the patient suffering from metastatic cancer, by far, by unnecessary and toxic doses of medications each with its own side effect profile, than by their quasi-religious continuation. Unfortunately, these straightforward tools are rarely used in the oncology clinic and almost never used sensibly or effectively. What has been lacking, however, is a cohesive system to bring these essential strategies to each cancer patient; as has a means 
for measuring the circadian organization of each cancer patient and her/his compliance with these simple behavioral and medical modulations and providing immediate positive feedback to the individual.

We, herein, outline evidence-based strategies and rationale for optimal circadian entrainment of patients with advanced cancer, followed by a proposal for the immediate application of our smartphone-based system for cancer patients that provides relevant circadian-related feedback and guidance. The system would include high-quality actigraphy that is capable of characterizing sleep, activity levels, light levels, and circadian indices in real time and provide feedback to the individual.

\section{Reduction of light exposure and blue light at bedtime} Epidemiological data strongly link exposure to nocturnal light pollution to the development of human cancer and its capacity to reset or delay sleep onsets in people as cause of the modern epidemic of sleep disorders and depression. ${ }^{41,42}$ Recent epidemiological research has strong evidence that nocturnal light is also linked to the ongoing metabolic syndrome epidemic. ${ }^{15,43}$ So there are several urgent public health problems associated with nocturnal exposure to artificial light. The problem is how to prevent excessive exposure to light at night. The standard advice is to use light blocking curtains, turn off lights in hallways, and remove light sources, especially televisions and computers from bedrooms. These measures do not help shift workers.

There is now a detailed understanding of the relationship between the light spectrum and the nocturnal production of melatonin, a surrogate variable for measuring whether the circadian clock is disrupted or intact. These investigations found that the short wavelengths of light or the blue region of the light spectrum suppress melatonin production, while the long wavelengths of light show no melatonin suppression; and it is the part of the visual spectrum where human visual acuity is at its peak. Unfortunately, very low levels of blue light can significantly reduce the pineal gland's nighttime production of melatonin in humans. ${ }^{44}$ These data have led to the development of blue blocker glasses and blue spectrum deficient light bulbs. Today some European countries are replacing conventional street lamp bulbs with blue spectrum deficient bulbs (European Commission, MEMO/09/368, Brussels, September 1, 2009). The impact of this public health measure should be closely monitored by cancer epidemiologists to quantitate the impact on the affected communities' cancer rates.

\section{Our simple solution}

Implementation of a smartphone-based system which includes a body worn light sensor and blue blocker glasses could help mitigate the circadian disruption due to poorly timed light exposure. High levels of ambient light detected at times that are inappropriate from a circadian perspective could trigger a real-time warning that the patient should don his/her blue blocker glasses. Absence of bright light at the appropriate time could trigger a suggestion that the patient go outdoors or use a light box designed for phototherapy.

\section{Improving sleep hygiene}

Satisfying sleep is an important sign of a robust and wellentrained endogenous circadian time structure and a healthy person. Surveys of sleep disturbances between different groups of cancer patients report prevalence rates ranging from $24 \%$ to $95 \%{ }^{45}$ Insomnia prevalence rates are high in cancer patients $^{46}$ and its onset coincides with the time at which cancer was actually diagnosed (from 6 months prediagnosis to 18 months postdiagnosis).

The causes of cancer patients' sleep disturbance are various and numerous. The symptoms of tumor invasion such as pain, dyspnoea, fatigue, nausea, and pruritus contribute to poor sleep. Patients sleep quality is adversely affected by the agents used to treat the cancer and other agents used to manage cancer or treatment related symptoms (narcotics, chemotherapy, neuroleptics, sympathomimetics, sedative/hypnotics, steroids, caffeine/nicotine, antidepressants, and diet supplements) and environmental factors (disturbing light and noise and/or extreme temperature in bedrooms). ${ }^{19}$

Numerous drugs are currently approved for the treatment of insomnia, but none have been tested for efficacy in cancer patients. ${ }^{46}$ The use of treatments for insomnia among cancer patients is wide spread. ${ }^{47}$ In a randomly selected sample of active patients and survivors, the prevalence of hypnotic medication use was $22.6 \%$. Factors associated with hypnotic medication use were older age, greater difficulty to fall asleep, stressful life events experienced over the past 6 months, higher levels of anxiety, past or current psychological difficulties, poorer role functioning, less severe urinary symptoms, larger use of opioids as well as past or current chemotherapy treatments. ${ }^{47}$

Assessing and, if need be, adjusting sleep hygiene is always the first step in correcting any sleep problem. Ranier Simeit et $\mathrm{al}^{48}$ described the benefit of a multimodal psychological sleep management program combining stimulus control, sleep hygiene, relaxation, and cognitive techniques. 
According to the National Sleep Foundation website, the elements of good sleep hygiene include the following:

- Maintain a regular wake and sleep pattern 7 days a week. Try to rise with the sun or expose your eyes to sunlight immediately upon daily awakening. Start the day with coffee or tea or some other similar stimulant.

- Breakfast should be taken after full awakening and after early morning exercise. It should be the heaviest and highest fat and carbohydrate meal of each day. The daily cortisol rhythm strongly supports a decent appetite within the first few hours after rising. Most daily calories should be consumed at breakfast.

- Avoid napping during the day, except at a fixed time and for 1 hour or less each day.

- Avoid stimulants such as caffeine, nicotine, and alcohol too close to bedtime.

- Exercise daily, each morning or midday, not close to bedtime as it will diminish sleepiness if done late in the day and it will make you sleepy in the evening, if done in the morning.

- The evening meal could be preceded and accompanied by alcohol if alcohol is desired (up to two drinks). The meal should be high in protein since the metabolism of protein is soporific.

- Food can be disruptive right before sleep. Stay away from large meals close to bedtime. Last meal $6 \mathrm{pm}$. Avoid alcohol after $6 \mathrm{pm}$ each evening.

- Ensure adequate exposure to natural light during each day, rain or shine.

- Establish a regular relaxing bedtime routine.

- Associate your bed with sleep.

\section{Our simple solution}

We propose that a smartphone-based app can be used to educate the patient about the importance of good sleep hygiene. When linked to an actigraph capable of accurately detecting naps, exercise, sleep schedule regularity, and light exposure, the app can help reinforce good hygiene by providing warnings and suggestions.

In addition, we propose using actigraphic technology that has been validated for use in sleep medicine. Besides allowing us to calculate real-time circadian indices, using actigraphic technology that has a validated agreement with the gold standard sleep measurement of polysomnography will allow us to include in the smartphone app a closed-loop feedback system for Cognitive Behavioral Therapy for Insomnia (CBT-I) which can be useful in treating sleeplessness in cancer patients. ${ }^{49}$ Monitored individuals can access video- based CBT-I directly through the smartphone; and passively recorded actigraphically measured sleep results provide feedback in the form of electronic coaching. Furthermore, a smartphone-based app can be used to keep track of the medications being used by cancer patient and the timing thereof. Whenever possible, it makes sense to defer administrations of medications which may have soporific side effects until the evening. This will reduce the propensity for daytime napping and enhance sleep consolidation at night.

This technology will also remind you daily of what to eat when, when to exercise, all of the above, and more temporal tuning advice.

\section{Mitigating daytime somnolence with proper medication avoidance, discontinuation, and when necessary, optimal timing}

Certain classes of pain medications have known side effects that include sleep disturbance, while others are known to cause drowsiness. Common sense would suggest that the former be avoided near bedtime and the latter should be delayed till nearer bedtime.

Pain medications are comprised of both long-acting ( $>12$ hours) and short-acting (4-6 hours) varieties. The inappropriate timing of essential pain medications causes as much or more cancer patient suffering than the inappropriate avoidance of adequate doses of pain medication when truly needed. Most cancer doctors and pain specialists know nothing about circadian organization and how it might affect a patient's optimal scheduling of various pain medications. Each individual cancer patient must have a careful and complete temporal history taken of the daily pattern of their pain and a rational chrono-pain plan devised and constantly updated.

At the same time there are extremely important generalities of circadian temporal organization of all human beings with or without metastatic cancer. The first 6 waking hours of each day, during the highest daily levels of the life-sustaining hormone cortisone are the hours when least pain medication is needed, and when appetite and well-being are maximum. If a cancer patient is unnecessarily "snowed" (stupefied) by an evening long-acting pain medication taken too late in the evening, the patient's normal circadian time structure can be destroyed for several days and the patient will have no chance to consume the calories necessary for health at the time of day when they go down most naturally and are most nourishing.

There are endless specific examples, but space does not permit going further, here. 


\section{Our simple solution}

Our proposed app would suggest timings for all prescribed medications based upon data, logic, and each patient's specific situation, as determined in the initial history taking process. Pain medications and sleep aids with short-acting soporific effects (like Benadryl 25 or $50 \mathrm{mg}$ ) will be recommended over longer acting ones. Another function of the app is the presentation of targeted educational videos which are immediately available through the app. An important class of videos would be about the timing of pain medication in general, and specific short videos making timing recommendations for each prescribed (or recommended over-the-counter) medication which affect sleep. One of the functions of the app is a medication diary. If a medication is taken at an inappropriate time in the circadian cycle, a reminder will be issued with an option to review the specific video recommendation for this medication.

\section{Making the life left more worth living}

Cancer patients feel lousy most of the time and their treatments can make them feel worse, before they make them feel better. Quality of life (QOL) issues often exacerbate treatment side effects and sometimes prevent appropriate treatment from being administered. The patient's inability to maintain optimal chemotherapy dosing, or interrupted treatment scheduling, or inability to complete a full course of treatment, leads to recurrence or progression of cancer, poorer response and shorter survival, thus leading to poorer QOL. Consequently, patient QOL can be directly related to patient survival.

Linking changes in a patient's circadian organization to their QOL is challenging. The clinical concept of QOL is that it is a sequential pathway initiated by a physiological disturbance that produces symptoms in patients that change their functional health, which in turn affects their perceptions of their general health, which in turn contributes directly to their perceptions of their overall QOL and level of satisfaction with their well-being. ${ }^{50,51}$ Furthermore, for each assessed QOL category there are a variety of different instruments. Physical domain assessment in one tool may measure symptoms, in a second the patient's ability to meet the challenges of daily life, or in a third adjustment to illness. ${ }^{52}$ There are no agreed definitions for various QOL attributes, which demonstrate that QOL is a multidimensional concept, within which each specific category has a range of unique attributes. ${ }^{52}$

\section{Circadian dysfunction affects global QOL}

The most intensive investigation on the relationship between baseline patients' circadian function and attributes of their QOL was conducted on the previously described late-stage lung cancer patients. Actigraphy data showed that these patients took many prolonged daytime naps, while suffering many episodes of prolonged awakening for prolonged spans of time each night. Similar findings have been found in early-stage breast cancer and metastatic colorectal cancer. ${ }^{35,36}$ These patients, like nearly all cancer patients, reported QOL outcomes that were significantly lower than population-based controls..$^{53}$ To summarize, the patients' responses to the EORTC QLQ C30 was that they were experiencing multiple symptoms and their capacity to meet the challenges of routine life was compromised. Their responses to the QLI tool which measures the satisfaction with the sources of their well-being, found that every patient's selfreport for the QLI health/functioning domain fell within the bottom quartile as compared to the general population scores, indicating a uniformly high level of dissatisfaction with their physical health, but their scores for the other three QLI domains, social/economic, family, and psychological/spiritual, were indistinguishable from those of the general population. These patients were emotionally and psychologically healthy, despite developing a lethal cancer that was causing a significant level of symptoms and adversely affecting their capacity to meet the challenges of normal life.

Among these patients there were statistically significant correlations between EORTC QLQ C30 scales of global health and circadian phase stability as reflected by the 24-hour autocorrelation $(r=0.53, P<0.01)$. The actigraphy parameter, night-day balance of time spent asleep and awake, was statistically significantly associated with the EORTC QLQ C30 functioning domains role $(r=0.56, P<0.01)$ and cognitive function ( $r=0.45, P=0.02$ ). Surprisingly there were no statistically significant relationships between any actigraphy parameter and role, social, and physical functioning. Outpatient fatigue levels are associated with diminished robustness of the circadian quotient ( $r=-0.40, P=0.04$ ), rhythm quotient $(r=-0.41, P=0.03)$, and night-day balance of time spent asleep $(r=-0.52, P<-0.01)$. So, patients reporting normal levels of fatigue had quantitatively robust day-night activity/sleep measurement differences. ${ }^{54}$ Similar results were found for pain where the less robust the day-night activity differences, the greater the pain. These data objectively verify that pain interferes with both sleep at night and activity during the day. Loss of appetite is likewise associated negatively with the actigraphy parameters night-day sleep balance.

The most interesting result was a negative one - the lack of a relationship between self-reported insomnia among outpatients and any objectively measured actigraphy parameter. The actigraphy data demonstrates that all of the patients' sleep was fragmented and unconsolidated and that these patients' 
self-report of their sleep quality, as measured by a validated sleep questionnaire, was indistinguishable from insomniacs. ${ }^{39}$ This disconnect between objective signs of very poor sleep and the perception of insomnia among advanced lung cancer is interesting. It seems that with cancer patients as with the healthy elderly, the perception of nocturnal sleeplessness and the reality of it are at odds.

Our measurements of the Ferrans/Powers QLI domains found that all four domains are significantly associated with the parameters "peak activity", and night-day sleep balance had a statistically significant relationship with all of the QLI domains of health/functioning ( $r=0.39, P=0.04)$, social/economic $(r=0.40, P<0.04)$, psychological/spiritual ( $r=0.45, P=0.02)$, and family $(r=0.33, P=0.10)$. The more robust the day/night difference between nocturnal and daytime activity levels, the better the patients score by each and every QLI domain. The health/functioning domain also had a statistically significant positive relationship with 24-hour autocorrelation, or day-to-day circadian stability of peak and trough activity timings $(r=0.45, P=0.02)$. In a multivariate analysis, we found that a robust activity/rest circadian rhythm is associated with greater patient satisfaction with health/ functioning, less fatigue, and better overall QOL. ${ }^{54}$

The many strong correlations between all Ferrans-Powers QLI domains suggest that a decaying circadian organization affects a patient's perception of their health and emotional/ psychological well-being before it actually reduces his/her capacity to fulfill these activities of normal life.

\section{Depression is associated with circadian} disruption caused by cancer

There is also accumulating data that actigraphy can be an effective tool to screen, diagnose, and manage patients with life shortening comorbid conditions like depression. Various investigations report prevalence rates of severe depression among cancer patients ranging from $6 \%$ to $42 \%$ at the time of diagnosis and treatment. ${ }^{19,55,56}$ While a change in mood is an expected reaction to a cancer diagnosis, patients with severe depression are much less likely to adhere to medical treatments, thereby diminishing the potential life prolonging benefits of therapy ${ }^{57-59}$ Among breast cancer patients, successful treatment for depression results in higher completion rates for adjuvant therapy and a corresponding lengthened lifespan. Unfortunately, depression is underrecognized and undertreated in cancer patients mostly because the symptoms of depression and cancer progression overlap..$^{60-62}$

A dysfunctional circadian function is a pathway shared between cancer- and tumor-free depressed patients. ${ }^{63} \mathrm{We}$ explored the relationship between cancer patients' self-report of depression using the Hospital Anxiety and Depressions Score (HADS) tool and their daily activity/rest circadian rhythm. Late-stage lung cancer patients were categorized as being depressed or not after reviewing their self-report on the HADS tool. Patients with actigraphic parameters of intact circadian function did not self-report as being depressed. While abnormal daily patterns of sleep-activity were associated with a self-report of depression, actigraphic data suggest the timing within the day (nighttime) of sleep is critical to the occurrence of depression. ${ }^{64}$ The next step in this research is to determine if there is a pattern of actigraphy results that is pathognomonic for clinical depression. Collecting self-reports of depression in a smartphone-based system along with indices of circadian entrainment derived from actigraphy would facilitate such research.

\section{Circadian dysfunction diminishes overall survival}

We now have data showing that patients with intact and robust circadian rhythms live longer than those with disrupted rhythms. Cortisol hormone concentration is a circadian regulated hormonal system, and breast and lung cancer patients with abnormally flattened cortisol levels have an elevated hazard of premature death..$^{65,66}$ Late-stage colorectal cancer patients with evidence of a relatively intact rest-activity circadian rhythm had median survivals of 21.6 months which is nearly double that of patients with disrupted function (11.9 months). ${ }^{67}$ Actigraphic data constitutes a robust and independent quantitative predictor of cancer patient outcomes. Actigraphy was used to measure sleep quality in late-stage breast cancer patient survivors whose potential lifespan is measured in years compared to months in late-stage colorectal cancer. Actigraphy data found that intact circadian rhythms in the form of sleep efficiency corresponded to a significant reduction in overall mortality (hazard ratio [HR], 0.96; 95\% confidence interval [CI], 0.94-0.98; $P<0.001)$ at median 6 years follow-up. This relationship remained significant after adjusting for other known prognostic factors (age, estrogen receptor status, cancer treatment, metastatic spread, cortisol levels, and depression). The investigators of this study interpreted their findings as showing that better sleep efficiency and less sleep disruption are significant independent prognostic factors in women with advanced breast cancer. ${ }^{68}$

\section{Our simple solution}

These results show that actigraphic data are effective predictors of cancer patient survival outcomes and this tool has 
the advantage of inexpensively and easily collecting such data from patients during their activities of normal daily life. We propose these circadian measures can act as a surrogate measure for QOL and can be collected passively from individuals via actigraphy, automatically analyzed, and appropriate advice provided - thereby allowing for interventions to be delivered in a timely way and their efficacy evaluated in an objective way.

\section{Exogenous melatonin therapy at bedtime}

Melatonin is very inexpensive and has a markedly low toxicity in human beings. ${ }^{42}$ There is extensive preclinical and animal data indicating it has antitumor effects. Theoretically, its role in synchronizing the peripheral clock oscillators may enhance a cancer patient's capacity to resist the deleterious effects of tumor progression and treatment in patients with treatment refractory disease where melatonin was the sole treatment (a wide spectrum of carcinoma patients were involved). Large doses in the range of 10-40 $\mathrm{mg} /$ day were used and survival rates were assessed after 1 year. The risk of death at 1 year was reduced by $34 \%$ and no adverse events were reported. In a second series of eight clinical trials where a once-a-day $20 \mathrm{mg}$ melatonin oral dosage was prescribed as an adjuvant therapy (in addition to chemotherapy or radiotherapy), a meta-analysis indicated that melatonin treated patients had a longer survival and a reduction in treatment related side effects such as thrombocytopenia, neurotoxicity, and fatigue. There are significant limitations: firstly, most trials were performed in the same center and the participants were not blinded and secondly, only two other studies were performed in different centers. ${ }^{69}$ Although the meta-analysis combined the sample size from eight trials, the total patient number is still relatively limited. Finally, the physiological and clinical assumptions for the $20 \mathrm{mg}$ dose have never been described in the literature. A recent study showed that a daily $20 \mathrm{mg}$ dose of melatonin improved the quality of sleep for patients with non-small-cell lung cancer and evening melatonin dosage timing enhanced the survival of patients given conventional chemotherapy.

\section{Our simple solution}

Keeping these limitations in mind, if melatonin therapy is to be included as part of a system to bolster circadian entrainment, a smartphone app can be used to cue the patient regarding the timing of administration (as well as for other medications) and using circadian measures can measure the efficacy of this intervention.
Properly timed exercise as a good way to tune time structure and feel better longer

The effect of exercise on circadian rhythms has been well established. ${ }^{70-73}$ Along with bright light therapy, proper timing of exercise can be used to maintain and support a normal rhythm. The overall effect of exercise on health and QOL cannot be doubted.

\section{Our simple solution}

We propose detection of levels of activity consistent with light exercise by a wrist-worn actigraph. The controlling smartphone app will be used to encourage mild exercise at the proper circadian time, during the first few hours after daily arising and with concurrent bright full spectrum light exposure.

\section{Optimally timed and constituted daily meals}

Likewise, proper timing of meals can support a normal circadian rhythm ${ }^{74}$ and meal content can also have an effect on sleep quality. ${ }^{75}$ In general, morning caloric fat and carbohydrate loading with evening high protein, low to moderate fat, and highcarbohydrate loading are supportive of a normal rhythm. ${ }^{76,77}$

\section{Our simple solution}

Light and exercise can be detected by the actigraph and the app can be used to conveniently enter meal content (dropdown selections of habitual foods will make this convenient) and timing. Automated messages from virtual circadian counselors can bolster compliance and answer questions.

\section{Components of the technology- assisted circadian "temporal tuning" system for cancer patients}

We propose a cohesive system employing all of the available inexpensive and benign tools for supporting a normal circadian rhythm. Such a system would include the following components:

1. A scientifically well-validated actigraph system that demonstrates acceptable agreement with gold-standard polysomnography in a sleep disrupted population.

2. The actigraph will include the ability to detect and record ambient light levels.

3. The actigraph will be capable of passive data transmission of activity and light levels to the individual's smartphone and on to the Cloud for appropriate real time data analysis and recommendations.

4. Detected light levels that present a circadian disadvantage will trigger an immediate alert and recommendation. 
5. This daily rhythm tuning system includes validated glasses that exclude blue wave length light, which is the wave length that interferes with the function of one's retinal clock.

6. In the absence of full spectrum outdoor bright light, the system will include an optional full spectrum light box.

7. The guts of the daily rhythm tuning system and origin of its real time recommendations is a smartphone app which integrates the individual's recorded data and sends it to the Cloud where real time day-to-day analysis is performed. These analyses trigger individualized appropriate recommendations, which are sent to the individual's smartphone; and a 24-hour summary is also sent each day at $12.00 \mathrm{am}$.

8. The smartphone app also includes immediate access to an internet-based CBT-I, which is a proven nonpharmaceutical based intervention to help sleeplessness in cancer patients.

9. The smartphone app provides informative tutorials and encouragement in the form of messages or a meaningful circadian index scale ranging from red to green.

10. While the intention is for the system to be as passive as possible, the smartphone platform provides a unique opportunity to collect an individual's subjective data as well (such as a pain rating scale).
Figure 1 demonstrates how intervention elements within the Temporal Tuning app might be organized. Real timing would be based on dawn/dusk in the patient's latitude and time of year.

We believe this program using simple behavioral modifications, actigraphic self-monitoring and appsuggested interventions for sleep quality/timing, activity quality/timing, nutrition quality/timing, medication timing (no soporifics and no pain medicines in the first half of the daily waking hours), morning light, morning exercise, morning caloric loading, and nightly proper sleep hygiene will allow cancer patients to remain synchronized with the world around them. Keeping them alert and awake when their loved ones are alert and awake by means of "temporal tuning" will allow them to remain connected, improve QOL, and survival rate.

\section{How the app works}

The critical sensor in this system would be an actigraph with validated sleep, nap, and exercise sensing technology linked wirelessly via Bluetooth to a smartphone, as shown in Figure 2.

The key to the success of the proposed system is seamless integration of all the proposed components such that the instructions, warnings, and recommendations are simple to follow while all necessary derived variables are also delivered
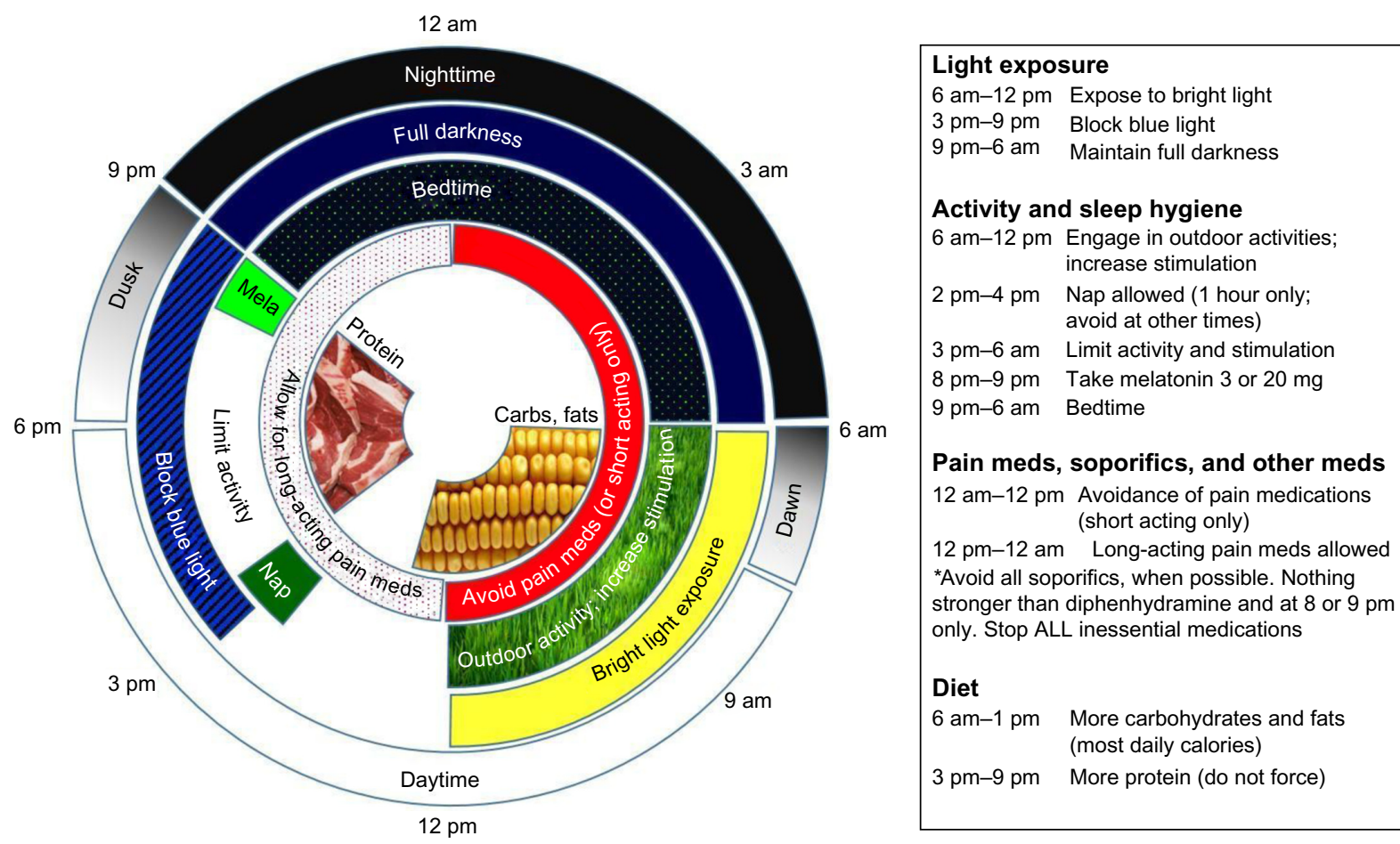

Pain meds, soporifics, and other meds

12 am-12 pm Avoidance of pain medications (short acting only)

12 pm-12 am Long-acting pain meds allowed *Avoid all soporifics, when possible. Nothing stronger than diphenhydramine and at 8 or $9 \mathrm{pm}$ only. Stop ALL inessential medications

Diet

6 am-1 pm More carbohydrates and fats (most daily calories)

3 pm-9 pm More protein (do not force)

Figure I Evidence-based optimal circadian timing of light exposure, sleep, activity, and diet. 


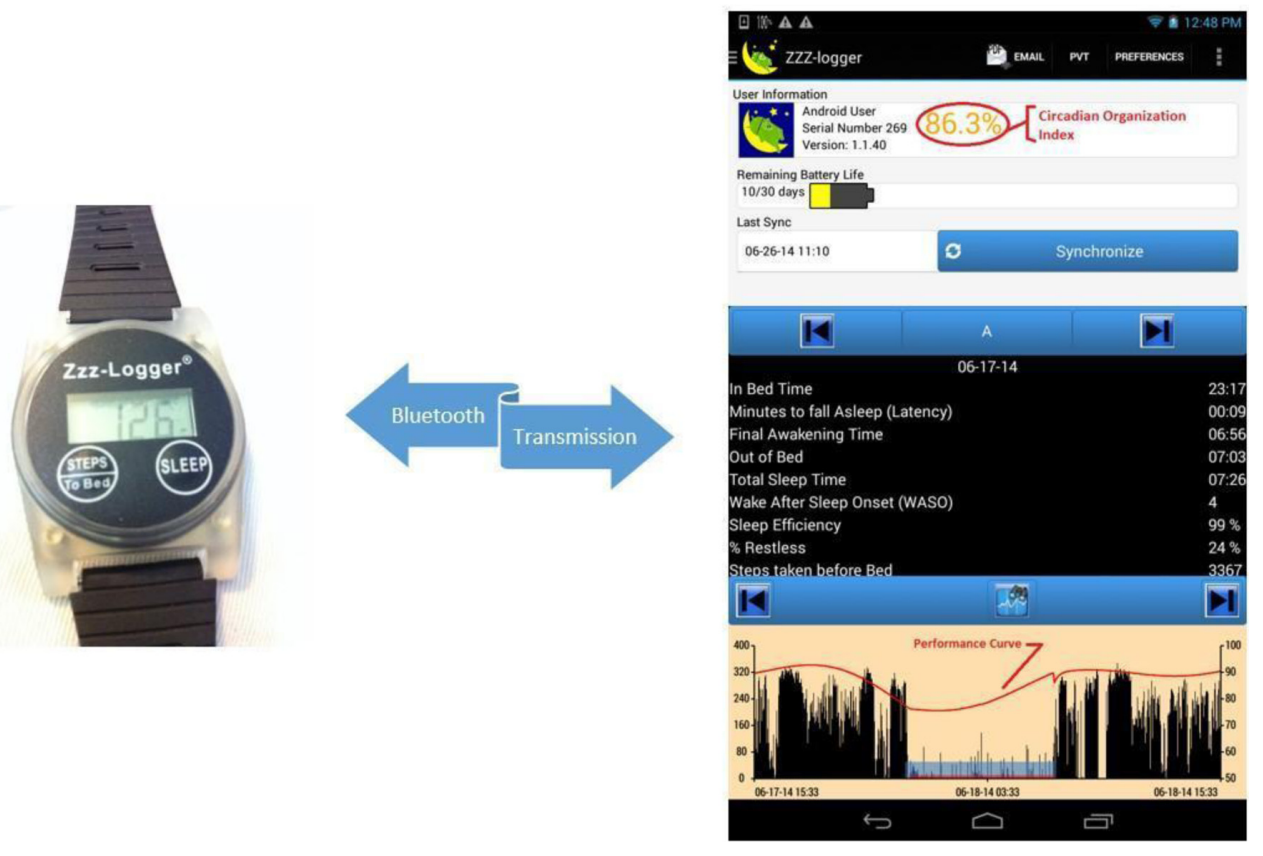

Figure 2 Actigraph-to-app schematic showing sleep results and a proposed index of circadian organization.

to the cancer patient or survivor in a comprehensive report. A typical interaction with the app looks like this:

9 am - Good Morning! Your sleep quality was fair last night but try to get to sleep 15 minutes earlier tonight, by $11 \mathrm{pm}$.

$9.20 \mathrm{am}$ - Thanks for entering your breakfast components in your food diary. Try to get some bright light exposure soon!

$10 \mathrm{am}$ - Yesterday you did 20 minutes of exercise at this time. How about some more exercise right now?

10.30 - Incoming call from your Temporal Tuning Coach. Answer?

$12.20 \mathrm{pm}$ - Thanks for entering your lunch components in your food diary.

$12.40 \mathrm{pm}$ - Thanks for entry in your medication diary. Try to take your next dose before $5 \mathrm{pm}$.

$6 \mathrm{pm}$ - Thank you for entering your dinner components in your food diary. Please refrain from snacking tonight if possible.

$7 \mathrm{pm}$ - Bright light detected. Please be sure you are wearing your Blue Blocker glasses.

$9.30 \mathrm{pm}$ - Are you getting ready for bed yet? Now might be a good time for a warm bath.

$11 \mathrm{pm}$ - Bedtime!

\section{Summary}

Developing public health strategies and clinical interventions that remediate disrupted human circadian function are an urgent opportunity in cancer medicine. We now have the ability to diminish or prevent cancer associated circadian disruption and to remediate circadian dysfunction in cancer patients undergoing chemotherapy. The strategies are evidence-based, well-recognized, simple, entirely noninvasive, safe, and inexpensive, if not free. They involve application of environmental/behavioral synchronizers such as meal timing, light exposure, good sleep hygiene, and the administration of agents that predictably affect and normalize and synchronize the cancer patient's circadian clocks with those of his/her family friends and broader society. This lessens the social isolation of cancer patients whether they are receiving treatment or supportive care. Specific circadian hormones without toxicity or meaningful cost, like melatonin, are also helpful. Our easy-to-use, smartphone-based technologies provide cancer patients and their physicians a cohesive, realtime, circadian-related information and feedback system to enhance cancer care quality and cancer patient QOL. Centers wishing to provide their cancer patients with this benefit add a unique safe, effective, virtually costless, and vital dimension to their cancer patients' lives.

\section{Acknowledgments}

The Gateway for Cancer Research; National Cancer Institute (1982-2000 RO1CA031635 grant, WJMH); VA (1989-2006 Merit Award, WJMH; 1989-2001 Merit Award, PAW); Life Extension Foundation; Oncology Analytics, Inc., Plantation, FL, USA; Ambulatory Monitoring, Inc., Ardsley, NY, USA. 


\section{Disclosure}

The authors report no conflict of interest in this work.

\section{References}

1. Watson J. Oxidants, antioxidants and the current incurability of metastatic cancers. Open Biol. 2013;3:120144.

2. Vogelstein B, Papadopoulos N, Velculescu V, Zhou S, Diaz LK Jr, Kinzler KW. Cancer genome landscapes. Science. 2013;339: 1546-1558.

3. Blau C, Liakopoulou E. Can we deconstruct cancer, one patient at a time? Trends Genet. 2013;29(1):6-10.

4. Haber D, Gray N, Baselga J. The evolving war on cancer. Cell. 2011;145(1):19-24.

5. Bozic I, Allen B, Nowak M. Dynamics of targeted cancer therapy. Trends Mol Med. 2012;18(6):311-316.

6. Joyner MJ, Prendergast FG. Chasing Mendel: five questions for personalized medicine. J Physiol. 2014;592:2381-2388.

7. Greaves M. Cancer; The Evolutionary Legacy. New York, NY: Oxford University Press; 2000:276.

8. Wagner A. The molecular origins of evolutionary innovations. Trends Genet. 2011;27(10):397-410.

9. Joyner MP. Chasing Mendel: five questions for personalized medicine. J Physiol. 2014;592:2381-2388.

10. Foster R, Wulff K. The rhythm of rest and excess. Nat Rev Neurosci. 2005;6:407-414.

11. Haimov I, Lavie P. Melatonin a chronobiotic and soporific hormone. Arch Gerontol Geriatr. 1997;24:167-173.

12. Hunter P. To sleep, perchance to live, Sleeping is vital for health, cognitive function, memory and long life. EMBO Rep. 2008;9:1070-1073.

13. Froy O. The circadian clock and metabolism. Clin Sci. 2011;120:65-72.

14. Rabstein S, Harth V, Justenhoven C, et al. Polymorphisms in circadian genes, night work and breast cancer: results from the GENICA study. Chronobiol Int. 2014;17:1-8.

15. Shanmugam V, Wafi A, 'al-Taweel N, Busselberg D. Disruption of circadian rhythm increases the risk of cancer, metabolic syndrome and cardiovascular disease. J Local Global Health Sci. 2013;209:3.

16. Sancar A, Lindsey-Boltz L, Gaddameedhi S, et al. Circadian clock, cancer, and chemotherapy. Biochemistry. Epub October 29, 2014.

17. Filipski E, et al. Disruption of circadian coordination accelerates malignant growth in mice. Pathol Biol (Paris). 2003;51:216-219.

18. Davis S, Mirick D, Stevens R. Night-shift work, light at night, and risk of breast cancer. J Natl Cancer Inst. 2001;93:1557-1562.

19. Rondanelli M, Faliva MA, Perna S, Antoniello N. Update on the role of melatonin in the prevention of cancer tumorigenesis and in the management of cancer correlates, such as sleep-wake and mood disturbances: review and remarks. Aging Clin Exp Res. 2013;25:499-510. doi:10.1007/s4050-013-0118-6.

20. Wood P, Yang X, Taber A, et al. Period 2 mutation accelerates ApcMin/+ tumorigenesis. Mol Cancer Res. 2008;6:1786-1793.

21. Zhao X, Cho H, Yu R, Atkins A, Downes M, Evans R. Nuclear receptors rock around the clock. EMBO Rep. 2014;15:518-528.

22. Yang X, Wood P, Oh E-Y, Du-Quiton J, Ansell C, Hrushesky W. Down regulation of circadian clock gene Period 2 accelerates breast cancer growth by altering its daily growth rhythm. Breast Cancer Res Treat. 2009; 117:423-431.

23. Yang W, Stockwell B. Inhibition of casein kinase I-epsilon induces cancer-cell-selective, PERIOD2-dependent growth arrest. Genome Biol. 2008;9:R92.

24. Van der Zee E, Havekes R, Barf RP, et al. Circadian time-place learning in mice depends on cry genes. Curr Biol. 2008;18:844-848.

25. Wyse C, Coogan A, Selman C, Hazlerigg D, Speakman JR. Association between mammalian lifespan and circadian free-running period: the circadian resonance hypothesis revisited. Biol Lett. 2010;6:696-698.

26. Claustrat B, Brun J, Chazot G. The basic physiology and pathophysiology of melatonin. Sleep Med Rev. 2005;9:11-24.
27. Savvidis CK. Circadian rhythm disruption in cancer biology. Mol Med. 2012;18:1249-1260.

28. Fonken L, Nelson R. Illuminating the deleterious effects of light at night. F1000 Med Rep. 2011;3:18. doi:10.3410/M3-18.

29. Reiter R, Tan D, Korkmaz A, et al. Light at night, chronodisruption, melatonin suppression, and cancer risk: a review. Crit Rev Oncog. 2007; 13:303-328.

30. Bhatti P, Mirick D, Davis S. Shift work and cancer. Am J Epidemiol. 2012;45:760-763.

31. Blask D, Dauchy R, Sauer L, Krause J, Brainard J. Light during darkness, melatonin suppression and cancer progression. Neuro Endocrinol Lett. 2002;22(Suppl 2):52-56.

32. Blask D, Brainard G, Dauchy R, et al. Melatonin depleted blood from premenopausal women exposed to light at night stimulates growth of human breast cancer xenografts in nude rats. Cancer Res. 2005;65:11174-11184

33. Dauchy R, Xiang S, Mao L, et al. Circadian and melatonin disruption by exposure to light at night drives intrinsic resistance to tamoxifen therapy in breast cancer. Cancer Res. 2014;74:1-12.

34. Blask DE, Hill SM, Dauchy RT, et al. Circadian regulation of molecular, dietary, and metabolic signaling mechanisms of human breast cancer growth by the nocturnal melatonin signal and the consequences of its disruption by light at night. J Pineal Res. 2011;51(3): 259-269

35. Berger A, Hertzog M, Geary C, Fischer P, Farr L. Circadian rhythms, symptoms, physical functioning, and body mass index in breast cancer survivors. J Cancer Surviv. 2012;6:305-314.

36. Innominato P, Focan F, Gorlia T, et al. Circadian rhythm in rest and activity: a biological correlate of quality of life and a predictor of survival in patients with metastatic colorectal cancer. Cancer Res. 2009;69: 4700-4707.

37. Grutsch J, Wood P, Du-Quiton J, et al. Validation of actigraphy to assess circadian organization and sleep quality in patients with advanced lung cancer. J Circadian Rhythms. 2011;9:4

38. Ohayon M, Carskadon M, Guilleminault CV. Meta-analysis of quantitative sleep parameters from childhood to old age in healthy individuals: developing normative sleep values across the human lifespan. Sleep. 2004;27:1255-1273.

39. Buysse D, Reynolds CI, Monk T, Berman S, Kupfer D. The Pittsburgh Quality Index: a new instrument for psychiatric practice and research. Psychiatry Res. 1989;28:193-213.

40. Littner M, Kushida C, Anderson W, et al. Practice parameters for the role of actigraphy in the study of sleep and circadian rhythms: an update for 2002. Sleep. 2003;26:337-341.

41. McClung C. Circadian genes, rhythms and the biology of mood disorders. Pharmacol Ther. 2007;114:222-232.

42. Wirz-Justice A. Temporal organization as a therapeutic target. Dialogues Clin Neurosci. 2012;14:335-337.

43. Nicolaides M, Charmandari E, Chrousos G, Kino T. Circadian endocrine rhythms: the hypothalamic-pituitary-adrenal axis and its actions. Ann NY Acad Sci. 2014;1318:71-80.

44. Stevens R, Brainard G, Blask D, Lockley S, Motta M. Breast cancer and circadian disruption from electric lighting in the modern world. CA Cancer J Clin. 2014;64:207-218.

45. Brzezinski A. Melatonin in humans. N Engl J Med. 1997;336: 186-195.

46. Savard J, Morin C. Insomnia in the context of cancer: a review of a neglected problem. J Clin Oncol. 2001;19:895-908.

47. Casault L, Savard J, Ivers H, Savard M, Simard S. Utilization of hypnotic medication in the context of cancer: predictors and frequency of use. Support Care Cancer. 2012;20:1203-1210.

48. Simeit R, Deck R, Conta-Marx B. Sleep management training for cancer patients with insomnia. Support Care Cancer. 2004;12:176-183.

49. Savard J, Ivers H, Savard MH, Morin CM. Is a video-based cognitive behavioral therapy for insomnia as efficacious as a professionally administered treatment in breast cancer? Results of a randomized controlled trial. Sleep. 2014;37(8):1305-1314. 
50. Bakas T, McLennon S, Carpenter J, et al. Systematic review of healthrelated quality of life models. Health Qual Life Outcomes. 2012;10:134. doi:10.1186/1477-7525-10-134.

51. Ferrans CE, Zerwic JJ, Wilbur JE, Larson JL. Conceptual model of health-related quality of life. J Nurs Scholarsh. 2005;37:336-342.

52. Ferrans CE. Differences in what quality-of-life instruments measure. J Natl Cancer Inst Monogr. 2007;(37):22-26.

53. McCabe R, Grutsch J, Nutakki S, Braun D, Markman M. Can quality of life assessments differentiate heterogeneous cancer patients? PLoS One. 2014;9(6):e99445.

54. Grutsch J, Ferrans C, Wood P, et al. The association of quality of life with potentially remediable disruptions of circadian sleep/ activity rhythms in patients with advanced lung cancer. BMC Cancer. 2011;11:193.

55. Badger T, Segrin C, Dorros S, Meek P, Lopez A. Depression and anxiety in women with breast cancer and their partners. Nurs Res. 2007;56(1):44-53.

56. Ng C, Boks M, Zaional N, De Wit N. The prevalence of pharmacotherapy of depression in cancer patients. J Affect Disord. 2011; $131: 1-7$.

57. Moussavi S, Chatterji S, Verdes E, Tandon A, Patel VU. Depression, chronic diseases, and decrements in health: results from the World Health Surveys. Lancet. 2007;370:851-858.

58. Stoudemire A, Thompson T. Medication non-compliance: systematic approaches to evaluation and intervention. Gen Hosp Psychiatry. 1983;5:233-239.

59. Montazeri A, Milroy R, Hole D, McEwen J, Gillis C. Anxiety and depression in patients with lung cancer before and after diagnosis: findings from a population in Glasgow, Scotland. J Epidemiol Community Health. 1998;52:203-204.

60. McDaniel J, Musselman D, Porter M, Reed M, Nemeroff C. Depression in patients with cancer, diagnosis, biology and treatment. Arch Gen Psychiatry. 1995;52:89-99.

61. Massie M. Prevalence of depression in patients with cancer. J Natl Cancer Inst Monogr. 2004;32:57-71.

62. Navari R, Brenner M, Wilson M. Treatment of depressive symptoms in patients with early stage breast cancer undergoing adjuvant therapy. Breast Cancer Res Treat. 2008;112:197-201.

63. Parker K, Bliwise D, Ribeiro M, et al. Sleep/wake patterns of individuals with advanced cancer measured by ambulatory polysomnography. J Clin Oncol. 2008;26:2464-2472.
64. Du-Quiton J, Wood P, Burch J, et al. Actigraphy assessment of daily sleep-activity pattern abnormalities reflects self-assessed depression and anxiety in outpatients with advanced non small cell, lung cancer. Psycho-Oncology. 2010;19:180-189.

65. Sephton S, Sapolsky R, Kraemer H, Spiegel D. Diurnal cortisol rhythm as a predictor of breast cancer survival. J Natl Cancer Inst. 2000;92:994-1000.

66. Sephton S, Lush E, Dedert E, et al. Diurnal cortisol rhythm as a predictor of lung cancer survival. Brain Behav Immun. 2013;30:S162-S170.

67. Dugue P-A, Innominato P, Karaoue A, et al. Wrist actimetry circadian rhythm as a robust predictor of colorectal cancer patients survival. Chronobiol Int. 2014;31(8):891-900.

68. Palesh O, Aldrige-Gerry A, Zeitzer J, et al. Actigraphy measured sleep disruption as a predictor of survival among women with advanced breast cancer. Sleep. 2014;37:837-842.

69. Wang Y, Jin B, Ai F, et al. The efficacy and safety of melatonin in concurrent chemotherapy or radiotherapy for solid tumors: a meta-analysis of randomized controlled trials. Cancer Chemother Pharmacol. 2012;69: 1213-1220.

70. Van Reeth O, Sturis J, Byrne MM, et al. Nocturnal exercise phase delays circadian rhythms of melatonin and thyrotropin secretion in normal men. Am J Physiol. 1994;266:E964-E974.

71. Schroder EA, Esser KA. Circadian rhythms, skeletal muscle molecular clocks, and exercise. Exerc Sport Sci Rev. 2013;41:224-229.

72. Baehr EK, Fogg LF, Eastman CI. Intermittent bright light and exercise to entrain human circadian rhythms to night work. Am J Physiol. 1999;277:R1598-R1604.

73. Eastman CI, Hoese EK, Youngstedt SD, Liu L. Phase-shifting human circadian rhythms with exercise during the night shift. Physiol Behav. 1995;58(6):1287-1291.

74. Oike H, Oishi K, Kobori M. Nutrients, clock genes, and chrononutrition. Curr Nutr Rep. 2014;3:204-212. eCollection 2014. Review.

75. Tanaka E, Yatsuya H, Uemura M, et al. Associations of protein, fat, and carbohydrate intakes with insomnia symptoms among middle-aged Japanese workers. J Epidemiol. 2013;23(2):132-138.

76. Grandner MA, Jackson N, Gerstner JR, Knutson KL. Dietary nutrients associated with short and long sleep duration. Data from a nationally representative sample. Appetite. 2013;64:71-80.

77. Afaghi A, O'Connor H, Chow CM. High-glycemic-index carbohydrate meals shorten sleep onset. Am J Clin Nutr. 2007;85(2):426-430. Erratum in: Am J Clin Nutr. 2007;86(3):809.
ChronoPhysiology and Therapy

\section{Publish your work in this journal}

ChronoPhysiology and Therapy is an international, peer-reviewed, open access journal focusing on research into the cyclic variations and rhythmicity in physiological processes in the body and the research and development and optimal timing of administration of therapeutic targets to achieve improved outcomes and quality of life for the patient. The

\section{Dovepress}

manuscript management system is completely online and includes a very quick and fair peer-review system. Visit http://www.dovepress.com/ testimonials.php to read real quotes from published authors. 